\title{
Autonomous Jerk Oscillator with Cosine Hyperbolic Nonlinearity: Analysis, FPGA Implementation, and Synchronization
}

\author{
Karthikeyan Rajagopal $\mathbb{D}$, ${ }^{1}$ Sifeu Takougang Kingni $\mathbb{D}^{2},{ }^{2}$ Gaetan Fautso Kuiate $\mathbb{D}^{3}{ }^{3}$ \\ Victor Kamdoum Tamba, ${ }^{4}$ and Viet-Thanh Pham ${ }^{5}{ }^{5}$ \\ ${ }^{1}$ Center for Nonlinear Dynamics, Defense University, Ethiopia \\ ${ }^{2}$ Department of Mechanical, Petroleum and Gas Engineering, Faculty of Mines and Petroleum Industries, University of Maroua, \\ P.O. Box 46, Maroua, Cameroon \\ ${ }^{3}$ Department of Physics, Higher Teacher Training College, The University of Bamenda, P.O. Box 39 Bamenda, Cameroon \\ ${ }^{4}$ Laboratory of Electronics and Signal Processing (LETS), Department of Physics, Faculty of Science, University of Dschang, \\ P. O. Box 67, Dschang, Cameroon \\ ${ }^{5}$ School of Electronics and Telecommunications, Hanoi University of Science and Technology, 01 Dai Co Viet, Hanoi, Vietnam \\ Correspondence should be addressed to Gaetan Fautso Kuiate; fautso_kuiate@yahoo.com
}

Received 22 May 2018; Revised 17 July 2018; Accepted 30 July 2018; Published 17 September 2018

Academic Editor: Dimitrios J. Frantzeskakis

Copyright (C) 2018 Karthikeyan Rajagopal et al. This is an open access article distributed under the Creative Commons Attribution License, which permits unrestricted use, distribution, and reproduction in any medium, provided the original work is properly cited.

\begin{abstract}
A two-parameter autonomous jerk oscillator with a cosine hyperbolic nonlinearity is proposed in this paper. Firstly, the stability of equilibrium points of proposed autonomous jerk oscillator is investigated by analyzing the characteristic equation and the existence of Hopf bifurcation is verified using one of the two parameters as a bifurcation parameter. By tuning its two parameters, various dynamical behaviors are found in the proposed autonomous jerk oscillator including periodic attractor, one-scroll chaotic attractor, and coexistence between chaotic and periodic attractors. The proposed autonomous jerk oscillator has period-doubling route to chaos with the variation of one of its parameters and reverse period-doubling route to chaos with the variation of its other parameter. The proposed autonomous jerk oscillator is modelled on Field Programmable Gate Array (FPGA) and the FPGA chip statistics and phase portraits are derived. The chaotic and coexistence of attractors generated in the proposed autonomous jerk oscillator are confirmed by FPGA implementation of the proposed autonomous jerk oscillator. A good qualitative agreement is illustrated between the numerical and FPGA results. Finally synchronization of unidirectional coupled identical proposed autonomous jerk oscillators is achieved using adaptive sliding mode control method.
\end{abstract}

\section{Introduction}

There has been a significant increase in studies of chaotic oscillators over the past decades because of their complex behaviors and their promising applications [1-9]. There are increasing works on chaos in jerk oscillators [10-12]. It is worth noting that if the scalar $x(t)$ is denoted as a physical variable at time $t$, the third derivative $\dddot{x}(t)$ presents the jerk [13]. Different jerk oscillators with chaos were summarized by Sprott [14]. By using Josephson junctions, Yalcin constructed a general jerk circuit with multiscroll and hypercube attractors [15]. Multiscroll attractors in jerk circuits were presented by Ma et al. [16]. An elegant chaotic oscillators based on jerk equation was implemented in a circuit where the nonlinearity was provided by a single diode [17]. A simple chaotic jerk circuit was used in a sound encryption scheme [18]. Especially, a three-dimensional novel jerk chaotic oscillator with two hyperbolic sinusoidal nonlinearities was reported in [19].

Researchers have shown an increased interest in multistability [20-26]. Multistability is associated with the presence 


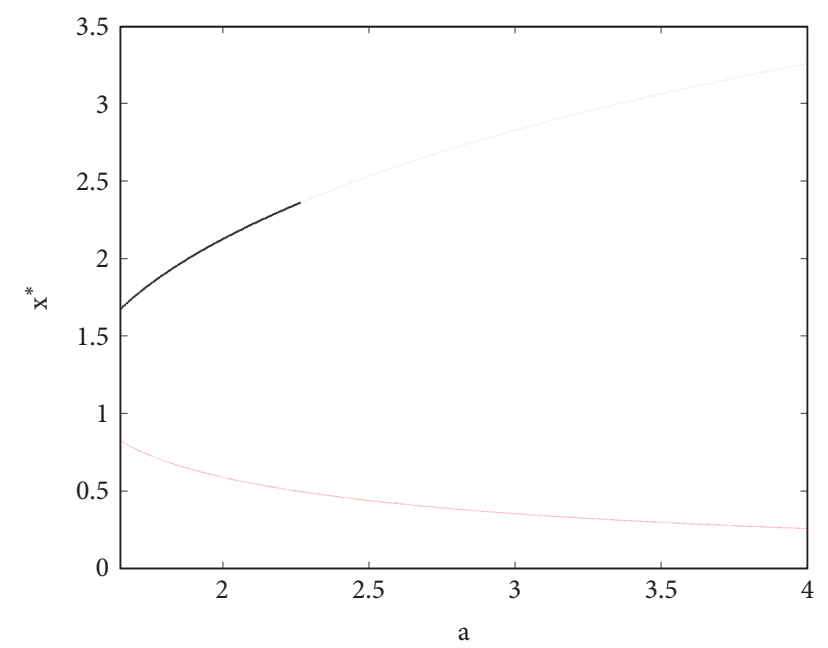

FIgURE 1: (Color online) Stability diagram of equilibrium points $E_{1,2}$ versus the parameter $a$ for $b=3$. Solid black lines indicate the stable branches and the dashed red lines the unstable branches.

of coexisting attractors with different initial conditions [20]. Jerk oscillators can exhibit multistability despite their simplicity [27-31]. Recently, Njitacke et al. discovered the coexistence of multiple attractors and crisis route to chaos in a chaotic jerk circuit [27] and a simple autonomous jerk oscillator with multiple attractors was introduced in [28]. Kengne et al. performed an analysis of a jerk circuit including a pair of semiconductor diodes connected in antiparallel and investigated its multiple attractors [29]. It is interesting that, by applying the diode bridge memristor into the original jerk circuit, authors proposed a novel hybrid diode based jerk circuit [30]. This work revealed that the hybrid diode based jerk circuit exhibits rich dynamical behaviors including multiple coexisting self-excited attractors. Derived from the autonomous jerk circuit, an autonomous memristor-based jerk circuit was constructed [31]. Interestingly, for the same values of system parameters, the coexistence of four different attractors was obtained in such a memristor-based jerk circuit.

Motived by published results related to jerk oscillators, some questions arose as to know, e.g., if a jerk oscillator with cosine hyperbolic nonlinearity can exhibit multistability or how such oscillator can synchronize. The aim of our work is to explore aspects of the unanswered questions. Our paper is structured as follows. Section 2 is devoted to the theoretical analysis of proposed autonomous jerk oscillator with a cosine hyperbolic term. FPGA implementation of proposed autonomous jerk oscillator is presented in Section 3. In Section 4, synchronization of unidirectional coupled jerk oscillators is studied by applying adaptive sliding mode control method. Finally, Section 5 concludes our work.

\section{Theoretical Analysis of Proposed Autonomous Jerk Oscillator}

In chapter three of "Elegant Chaos: Algebraically Simple flow" book published in 2010, Sprott proposed a list of sixteen autonomous chaotic jerk oscillators with different nonlinearities called memory oscillators [14]. The nonlinearities of memory oscillators include quadratic, cubic, quintic, absolute, maximum, sign, exponential, sine, and tangent hyperbolic functions. Inspired by [14], in this work we introduce an autonomous jerk oscillator with a cosine hyperbolic nonlinearity described by

$$
\begin{aligned}
& \frac{d x}{d t}=z, \\
& \frac{d y}{d t}=a x-y-b z-\cosh (x), \\
& \frac{d z}{d t}=y,
\end{aligned}
$$

where $x, y, z$ are state variables of the oscillator and $a, b$ two positive parameters. System (1a), (1b), and (1c) can be converted to a jerk oscillator, as follows:

$$
\frac{d^{3} x}{d t^{3}}=-\frac{d^{2} x}{d t^{2}}-b \frac{d x}{d t}+a x-\cosh (x) .
$$

System (1a), (1b), and (1c) is dissipative because $\nabla V=$ $\partial(d x / d t) / \partial x+\partial(d y / d t) / \partial y+\partial(d z / d t) / \partial z=-1$. The equilibrium points of system (1a), (1b), and (1c) are obtained by solving $d x / d t=0, d y / d t=0, d z / d t=0$, which gives

$$
\begin{aligned}
y^{*} & =z^{*}=0, \\
a x^{*}-\cosh \left(x^{*}\right) & =0 .
\end{aligned}
$$

Equation (3b) cannot be solved analytically. We thus use the Newton-Raphson method to find the value of $x^{*}$. Depending on the value of parameter $a$, (3b) presents no roots for $0 \leq$ $a<1.509$ and one root $\left(x^{*}\right)$ for $a \geq 1.509$. Therefore, for $0 \leq a<1.509$, system (1a), (1b), and (1c) has no equilibrium points while, for $a \geq 1.509$, it has two equilibrium points $E_{1}=$ $\left(x_{1}^{*}, 0,0\right)$ and $E_{2}=\left(x_{2}^{*}, 0,0\right)$ with $x_{1}^{*}>x_{2}^{*}$ (see Figure 1 ). The characteristic equation evaluated at the equilibrium point $E=\left(x^{*}, 0,0\right)$ is

$$
\lambda^{3}+\lambda^{2}+b \lambda-a+\sinh \left(x^{*}\right)=0 .
$$

According to the Routh-Hurwitz criterion, the real parts of all the roots $\lambda$ of (4) are negative if and only if

$$
\begin{aligned}
-a+\sinh \left(x^{*}\right) & >0 \\
a+b-\sinh \left(x^{*}\right) & >0
\end{aligned}
$$

The stability analysis of equilibrium point $E_{1}$ as function of the parameter $a$ of system (1a), (1b), and (1c) is depicted in Figure 1.

From Figure 1, one can notice that equilibrium point $E_{2}$ is always unstable for $1.65 \leq a \leq 4$, whereas the equilibrium point $E_{1}$ is stable for $1.65 \leq a \leq 2.269$ and unstable for $a>2.269$. Since the equilibrium points $E_{1}$ is stable for $1.65 \leq$ $a \leq 2.269$ but it changes stability properties at $a \approx 2.269$, system (1a), (1b), and (1c) can exhibit a Hopf bifurcation from equilibrium point $E_{1}$ when the parameter $a$ varies. 


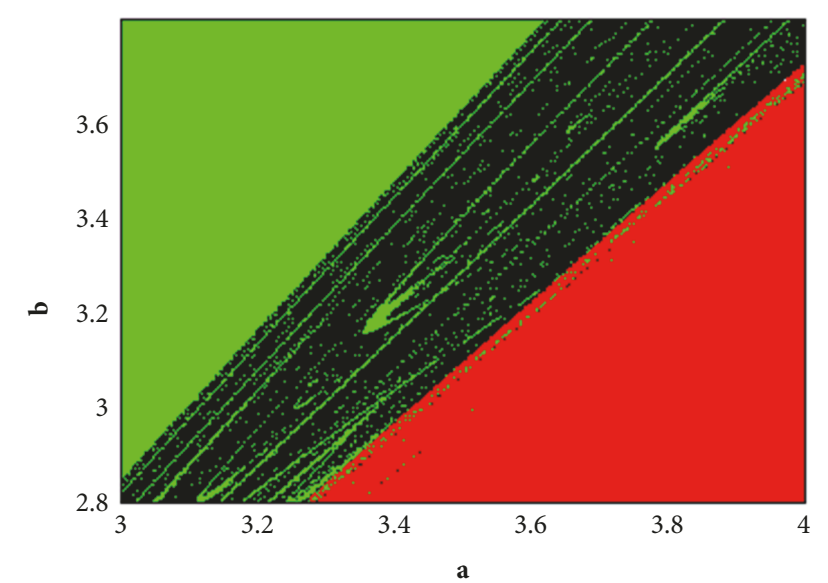

FIgURE 2: (Color online) Two-parameter $(a, b)$ bifurcation diagram depicting the dynamical behaviors of system (1a), (1b), and (1c). Unbounded orbits are in red; periodic oscillations are in green and chaos is in black.

Theorem 1. System (1a), (1b), and (1c) undergoes a Hopf bifurcation at the equilibrium point $E_{1}$ if $b>0$ and the parameter $a$ passes through the critical value $a_{H}=-b+\sinh \left(x_{1}^{*}\right)$.

Proof. Substituting $\lambda=i \omega(\omega>0)$ into (4) and separating real and imaginary parts, we obtain

$$
\begin{aligned}
& \omega=\omega_{0}=\sqrt{b}, \\
& a=a_{H}=-b+\sinh \left(x_{1}^{*}\right) .
\end{aligned}
$$

Differentiating both sides of (4) with respect to $a$, we can obtain

$$
3 \lambda^{2} \frac{d \lambda}{d a}+2 \lambda \frac{d \lambda}{d a}+b \frac{d \lambda}{d a}-1=0
$$

and

$$
\frac{d \lambda}{d a}=\frac{1}{3 \lambda^{2}+2 \lambda+b}
$$

then

$$
\operatorname{Re}\left(\left.\frac{d \lambda}{d a}\right|_{a=a_{H}, \lambda=i \omega_{0}}\right)=\frac{-1}{2(b+1)} \neq 0 .
$$

All the conditions for Hopf bifurcation to occur are met; therefore system (1a), (1b), and (1c) undergoes a Hopf bifurcation at $E_{1}$ when $a_{H}=-b+\sinh \left(x_{1}^{*}\right)$ and periodic solutions will exist in a neighbourhood of the point $a_{H}$ (provided that $b>0$ holds). If $b=3$, the critical value is $a=a_{H} \approx 2.269$. For $a=2.2<a_{H}$, the trajectories of jerk system (1a), (1b), and (1c) converge to the equilibrium point $E_{1}$ whereas, for $a=2.5>a_{H}$, system (1a), (1b), and (1c) exhibits a limit cycle (not shown).

We plot in Figure 2 a two-parameter $(a, b)$ bifurcation diagram depicting the dynamical behaviors of system (1a), (1b), and (1c).

Figure 2 indicated that system (1a), (1b), and (1c) can display unbounded orbits and periodic and chaotic behaviors. In
Figure 3, we firstly fix $b=3$ and plot the bifurcation diagram with respect to $a$ and the related largest Lyapunov exponent.

The bifurcation diagram of the output $x(t)$ in Figure 2(a) shows that the trajectories of system (1a), (1b), and (1c) converge to the equilibrium point $E_{1}$ up to $a \approx 2.269$ where a Hopf bifurcation occurs followed by a limit cycle motion (i.e., period-1 oscillations) and period-doubling to chaos interspersed with periodic windows. The largest Lyapunov exponent shown in Figure 3(b) confirms the chaotic behavior found in Figure 3(a). The chaotic behavior is illustrated in Figure 4 for a specific value of parameter $a$.

The trajectories of chaotic attractor are swirling around one of the two equilibrium points in Figure 4. This is a signature of one-scroll chaotic attractor.

Secondly, for $a=3.3$, sample results showing the bifurcation diagram versus $b$ and the related plot of Largest Lyapunov exponent are provided in Figure 5.

By varying parameter $b$ from 2.87 to 3.8 , the bifurcation diagram of the output $x(t)$ in Figure 5(a) displays chaotic behavior interspersed with periodic windows. For $b>3.18$, period-12-oscillations, period-6-oscillations, and period-3oscillations are found, respectively, up to $b<3.2012$ followed by reverse period-doubling to chaos interspersed with periodic windows. By varying parameter $b$ from 3.8 to 2.87 [see red dots of Figure 5(a)], the output $x(t)$ displays the same dynamical behaviors as shown by black dots of Figure 5(a) in the ranges $2.87 \leq b \leq 3.182$ and $3.2012 \leq b \leq 3.8$, while, in the range $3.182<b<3.2012$, the output $x(t)$ presents chaotic behavior. By comparing black dots of Figure 5(a) and red dots of Figure 5(a), one can notice that system (1a), (1b), and (1c) displays coexistence of period-6-oscillations and chaotic attractors in the range $3.182<b<3.188$ and coexistence of period-3-oscillations and chaotic attractors in the range $3.188 \leq b<3.2012$. The largest Lyapunov exponent shown in Figure 5(b) confirms the chaotic behavior found in Figure 5(a). Figure 6 depicts the phase portraits of coexisting attractors found in Figure 5(a) for specific values of parameter $b$.

For $b=3.185$, system (1a), (1b), and (1c) can exhibit either one-scroll chaotic attractor or period-6-oscillations depending on initial conditions as shown in Figure 6(a), while, in Figure 6(b) for $b=3.189$, system (1a), (1b), and (1c) can exhibit either one-scroll chaotic attractor or period-3oscillations depending on initial conditions. Figure 7 presents the basin of attraction of system (1a), (1b), and (1c) in the plane $y=0$ for $a=3.3$ and $b=3.189$.

In Figure 7, red, green, and black regions contain initial conditions that lead to unbounded orbits, periodic and chaotic attractors, respectively. One can see from the basin of attraction that the possibility of occurrence of unbounded orbits is greater than the ones of periodic and chaotic attractors.

\section{FPGA Implementation of Proposed Autonomous Jerk Oscillator}

There have been many literatures about implementation of chaotic oscillators using FPGA like FPGA based multiscroll attractor discussed in [32-39], digital chaotic oscillators and 


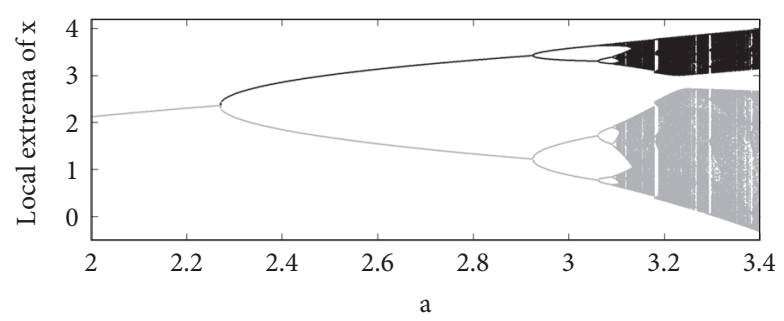

(a)

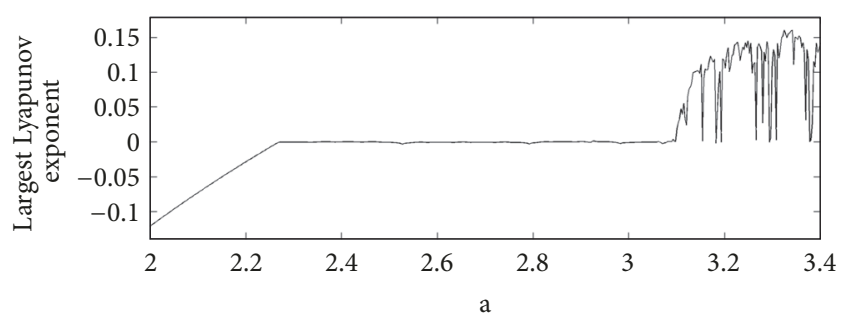

(b)

FIGURE 3: The bifurcation diagrams depicting the local maxima (black dots) and local minima (gray dots) of $x(t)$ (a) and the largest Lyapunov exponents (b) versus the parameter $a$ for $b=3$.
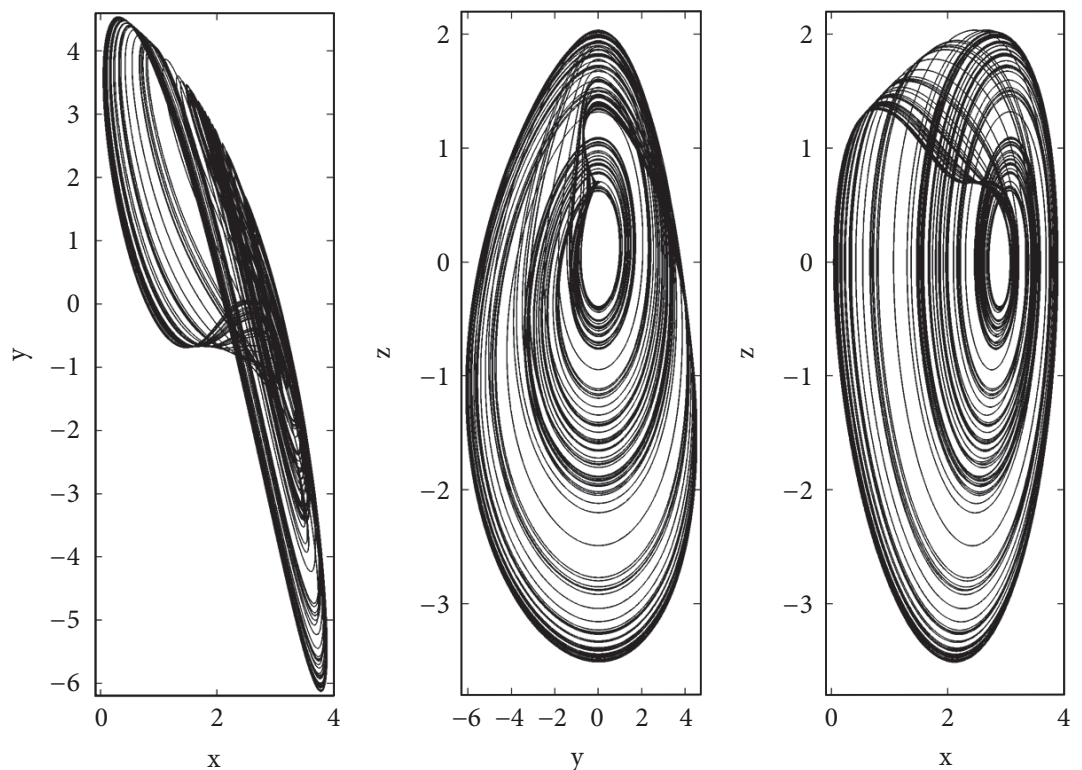

FIGURE 4: 2D phase portrait of system (1a), (1b), and (1c) for $a=3.3$ and $b=3$. The initial conditions are $(\mathrm{x}(0), \mathrm{y}(0), \mathrm{z}(0))=(1,0,0.5)$.

its FPGA model [36, 40], FPGA based four-wing chaotic attractor [37], and image cryptography with chaotic ciphers and its FPGA implementations and FPGA implemented memristor-based chaotic attractors [41]. Hyperchaotic oscillators with complex mathematical models are also implemented using FPGA and show that the FPGA performs better in power efficiency $[42,43]$. More complex synchronization algorithms are also implemented in FPGA such as synchronization of Ikeda oscillators [44], genetically optimized PID and sliding mode control based synchronizations [45, 46], and adaptive sliding mode synchronization [47]. These FPGA realizations are mainly based on hardware-software cosimulations [45-47] and with experimental analysis [44]. FPGA are also used in implementing various chaos-based encryption schemes like chaos-based pseudorandom number generator [48]. In this section we propose to implement the proposed autonomous jerk oscillator using FPGA. The major task in this implementation is to decide the type of numerical method to solve the dynamical system. We choose the forward Euler method [40] to solve the proposed autonomous jerk oscillator and the set of discretized system equations is given as follows:

$$
\begin{aligned}
& x_{k+1}=x_{k}+h\left[z_{k-1}\right], \\
& y_{k+1}=y_{k}+h\left[a x_{k-1}-y_{k-1}-b z_{k-1}-\cosh \left(x_{k-1}\right)\right], \\
& z_{k+1}=z_{k}+h\left[y_{k-1}\right],
\end{aligned}
$$

where $a, b$ are the system parameters and $h$ is the step size for the discrete numerical solution. To implement the hyperbolic cosine function, we use the Taylor series expansion with the first three polynomials as

$$
\cosh (x)=1+\frac{x^{2}}{2 !}+\frac{x^{4}}{4 !}+\frac{x^{6}}{6 !} .
$$

To achieve the accuracy we select the step size $h=0.001$ and as per the IEEE754 standards, we use the calculation of 32bit finite integer instead of floating-point operation. By using the arithmetic operations multiplying, adding or subtracting 


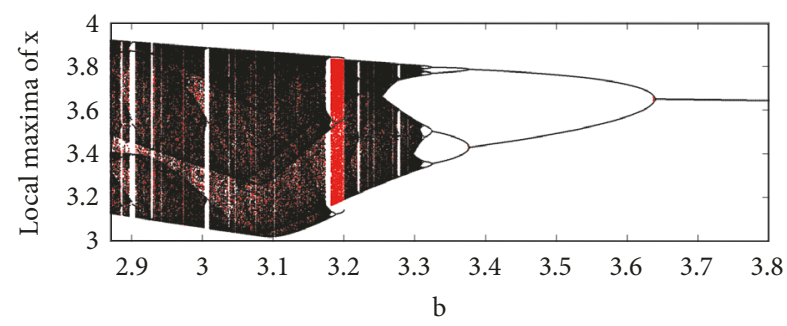

(a)

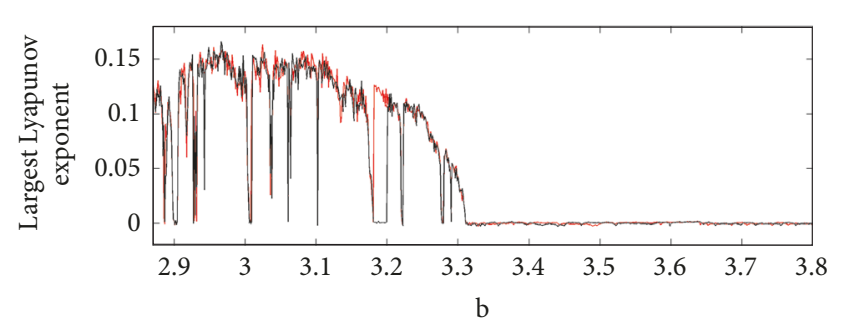

(b)

FIGURE 5: (Color online) The bifurcation diagrams depicting the local maxima of $x(t)$ (a) and the largest Lyapunov exponents (b) versus the parameter $b$ for $a=3.3$. In the graph (a), two sets of data corresponding, respectively, to increasing (black dot) and decreasing (red dot) values of $b$ are superimposed. Largest Lyapunov exponents are obtained by scanning parameter $b$ upwards (black line) and downwards (red line).
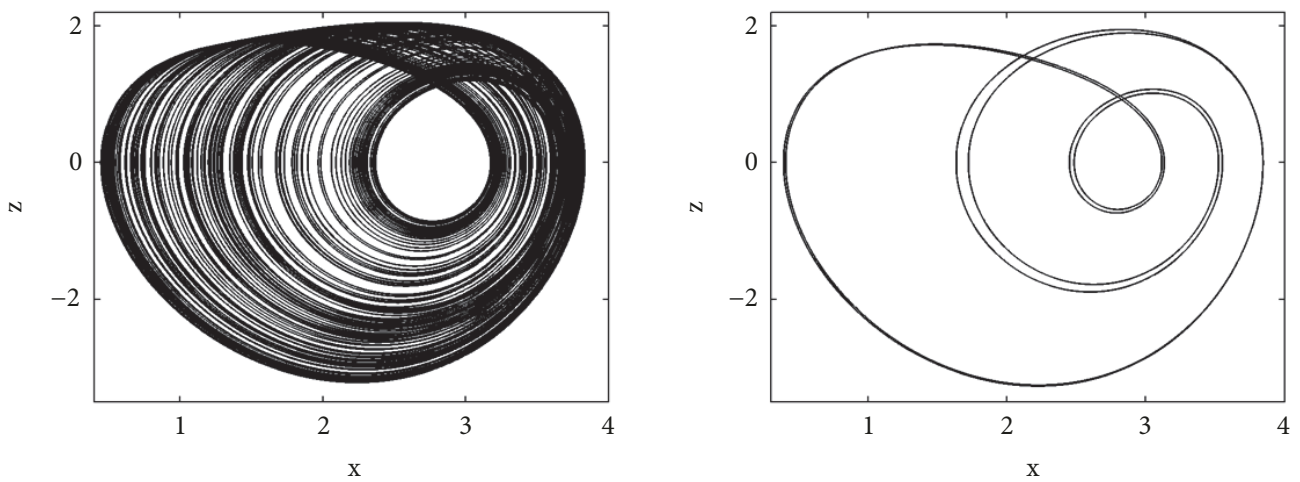

(a)
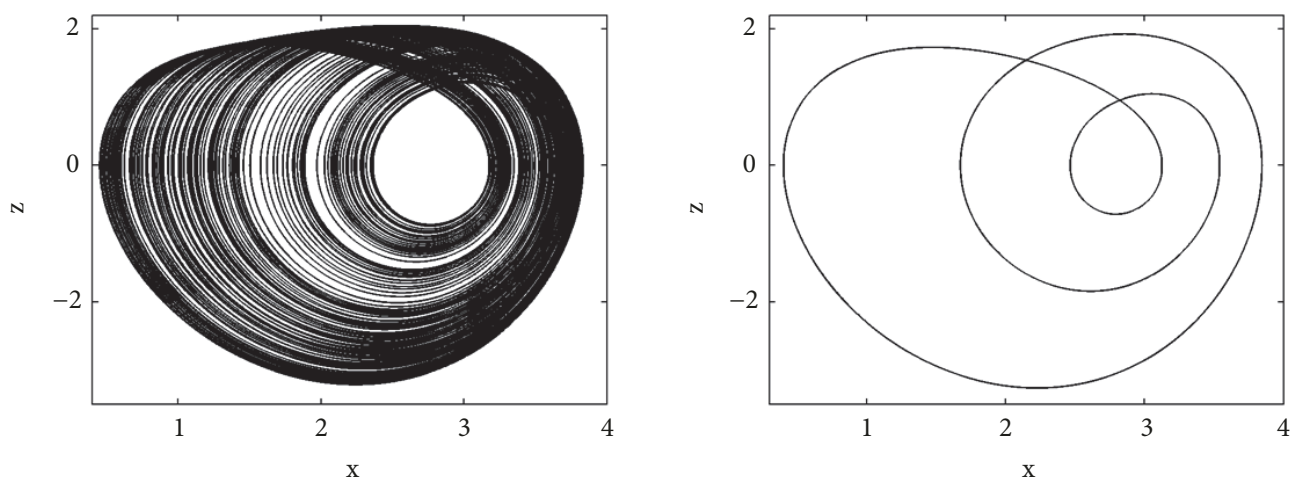

(b)

FIGURE 6: Phase portraits in the plane $(x, z)$ for specific values of parameter $b$ : (a) $b=3.185$ and (b) $b=3.189$. The initial conditions are $(\mathrm{x}(0), \mathrm{y}(0), \mathrm{z}(0))=(1.0,0,0.5)$ in left column and $(\mathrm{x}(0), \mathrm{y}(0), \mathrm{z}(0))=(3.0,0,0.5)$ in right column. The other parameter value is $a=3.3$.

the system (8a), (8b), and (8c) is implemented and the initial conditions for the next iteration can be obtained. By repeating this process of iteration we can derive the discrete chaotic system. The absolute value operation only needs to set the first bit of the 32-bit integer to 0 . The discrete state equations are implemented using hardware and software cosimulations and the needed basic arithmetic operators are implemented using the Xilinx system generator toolkit. We used the Kintex 7 chipset (xc7k160tfbg)) for the cosimulations and Matlab Simulink is used to plot the phase plots. The latency of the arithmetic blocks is kept at 3 and the maximum clock frequency of the FPGA used is $437 \mathrm{MHz}$. The register-transfer level (RTL) schematic of hyperbolic cosine function given by (9) is not shown while the RTL schematic of proposed autonomous jerk oscillator is shown in Figure 8.

Table 1 shows the resources utilized by the proposed autonomous jerk oscillator for implementing in Kintex-7.

Figure 9 shows the 2D phase portraits of the proposed autonomous jerk oscillator.

Figure 10 shows the coexisting attractors exhibited by the FPGA implemented proposed autonomous jerk oscillator for two different initial conditions.

One-scroll chaotic attractor and coexistence of attractors are clearly seen from Figures 9 and 10, respectively. By 


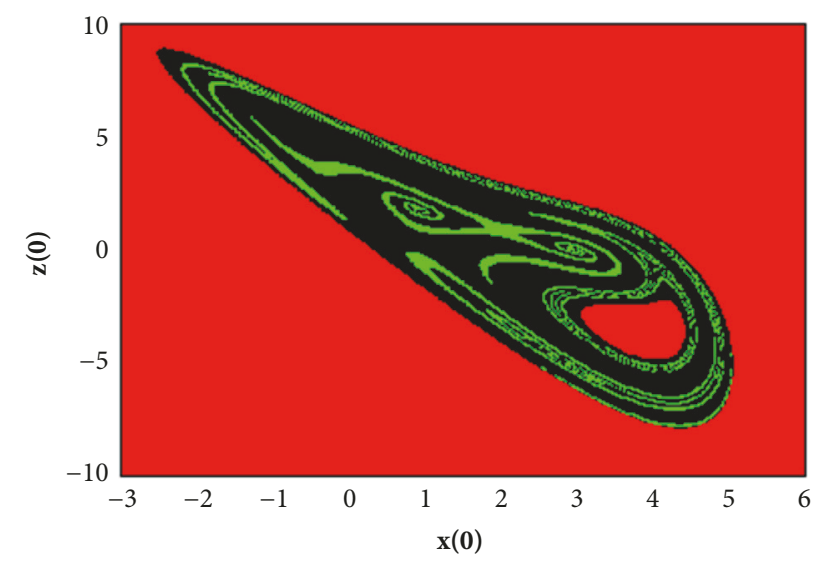

Figure 7: (Color online) Cross section of the basin of attraction of system (1a), (1b), and (1c) in the x-z-plane at $y=0$ for $a=3.3$ and $b=3.189$. Initial conditions in the black region lead to unbounded orbits, those in the green region lead to the periodic attractor and those in the red region lead to the strange attractor.

TABLE 1: Resources consumed by the FPGA implemented of proposed autonomous jerk oscillator.

\begin{tabular}{lccc}
\hline Resource & Utilization & Available & Utilization \% \\
\hline LUT & 880 & 101400 & 0.87 \\
FF & 522 & 202800 & 0.26 \\
DSP & 20 & 600 & 3.33 \\
IO & 97 & 285 & 34.04 \\
BUFG & 1 & 32 & 3.13 \\
\hline
\end{tabular}

comparing Figures 9 and 10 with Figures 9 and 10, it can be noticed that there is a good qualitative agreement between the numerical simulations and FPGA results.

\section{Synchronization of Unidirectional Coupled Proposed Autonomous Jerk Oscillators Using Adaptive Sliding Mode Control Method}

Here we present synchronization results of coupled chaotic proposed jerk oscillators in order to promote chaos-based synchronization designs of this type of proposed jerk oscillator. Synchronization of chaotic systems has applications in secure communication and cryptography. The highly sensitive nature of chaotic oscillators to initial conditions makes it difficult to synchronize the oscillators with uncertainties and disturbance. Some well-known ways to synchronize chaotic oscillators are using active control method $[49,50]$ adaptive control method [51, 52], extended back stepping control method [53, 54], sliding mode control method [55, 56], adaptive sliding mode control method [56-59], etc. Sliding mode control method is applied to provide robustness in the face of internal and external disturbances. In section, we use adaptive sliding mode control method for synchronization of unidirectional coupled identical proposed autonomous jerk oscillators using adaptive sliding mode control method.
4.1. Problem Statement. Let the generalized master system be defined as

$$
\dot{x}=f(x)+F(x) c
$$

and the slave system as

$$
\dot{y}=g(y)+G(y) d+u(t)
$$

where $f(x), g(y)$ are $n \times 1$ row vector and $F(x), G(y)$ are $m \times n$ matrix elements of the master and slave systems, respectively, $c, d$ are the unknown parameters of the systems, and $u(t)$ is the controller to synchronize the systems. The control objective is to synchronize the slave system with initial condition $y(0)$ with master system of initial condition $x(0)$ such that the synchronization errors (12) approaches zero.

$$
\lim _{t \rightarrow \infty} e_{i}=y_{i}-x_{i} .
$$

The sliding mode controller design for synchronizing the two systems involves selection of sliding surface for the desired dynamics and designing the reaching law such that any point on the phase space is brought to the sliding surface in the presence of uncertainties.

Let us define the proportional integral sliding surface [60] as

$$
s_{i}=e_{i}+K \int e_{i}(\tau) d \tau
$$

where $K$ is the proportional constant vector $\left[k_{1}, k_{2}, k_{3}\right]$.

The first derivative of the sliding surface is derived as

$$
\dot{s}_{i}=\dot{e}_{i}+K e_{i} .
$$

For the existence of the sliding mode, it is necessary and sufficient that the sliding surface and its first derivative should be equal to zero. The error dynamics can be derived as

$$
\dot{e}=g\left(x_{s}\right)+G\left(x_{s}\right) d+u(t)-f\left(x_{m}\right)-F\left(x_{m}\right) c .
$$

In order to avoid the chattering phenomenon caused by discontinuous control signals, the adaptive controller used to synchronize the master and slave system is chosen as

$$
\begin{aligned}
u(t)= & -g\left(x_{s}\right)-G\left(x_{s}\right) \widehat{d}+f\left(x_{m}\right)-F\left(x_{m}\right) \widehat{c}-k e \\
& -\eta \tanh (s)-\rho s
\end{aligned}
$$

where $k, \eta, \rho$ are positive gain values, $\widehat{c}, \widehat{d}$ are parameter estimates of master and slave systems, and $s$ is the sliding surface.

Using (16) in (15), the error dynamics simplifies to

$$
\dot{e}=G\left(x_{s}\right)[d-\widehat{d}]-F\left(x_{m}\right)[c-\widehat{c}]-\eta \tanh (s)-\rho s .
$$

The stability of the proposed controller can be analyzed using the Lyapunov candidate function:

$$
V=\frac{1}{2} s^{2}+\frac{1}{2}(d-\widehat{d})^{2}+\frac{1}{2}(c-\widehat{c})^{2} .
$$




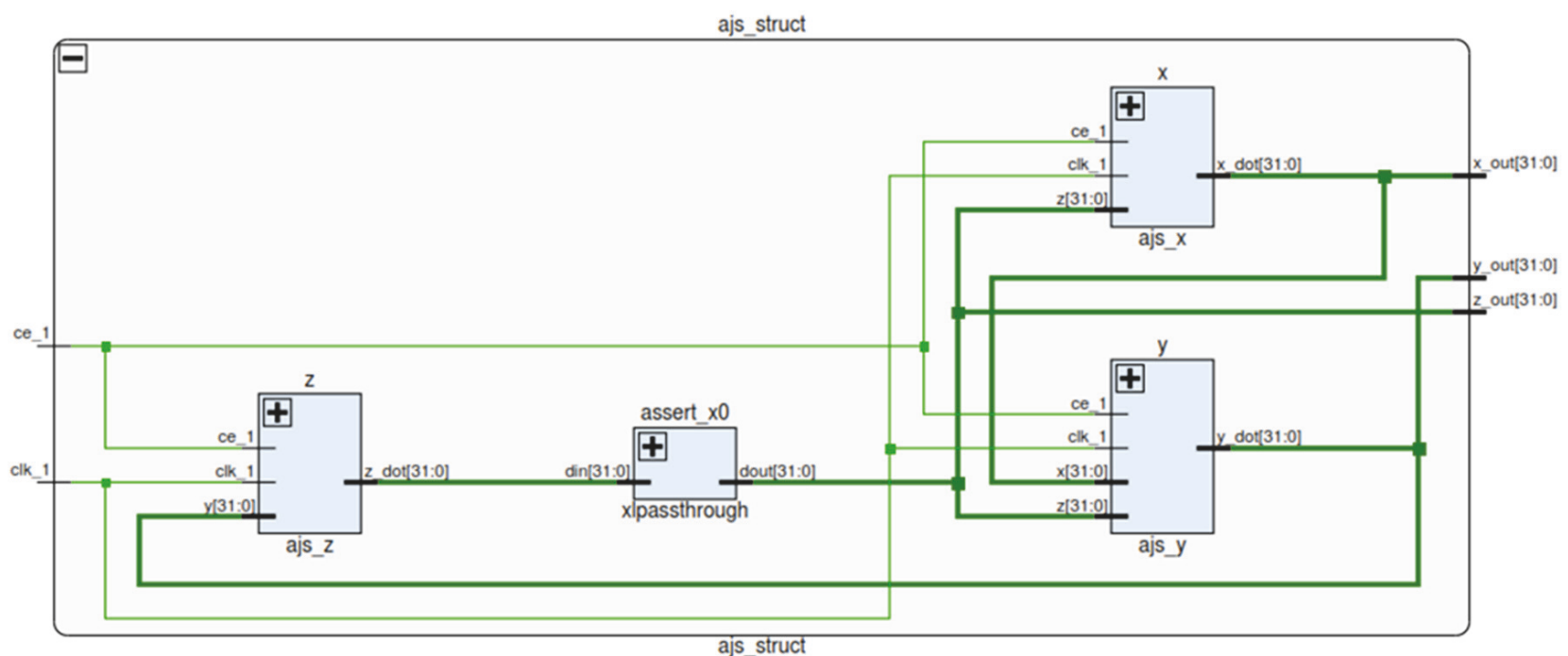

(a)

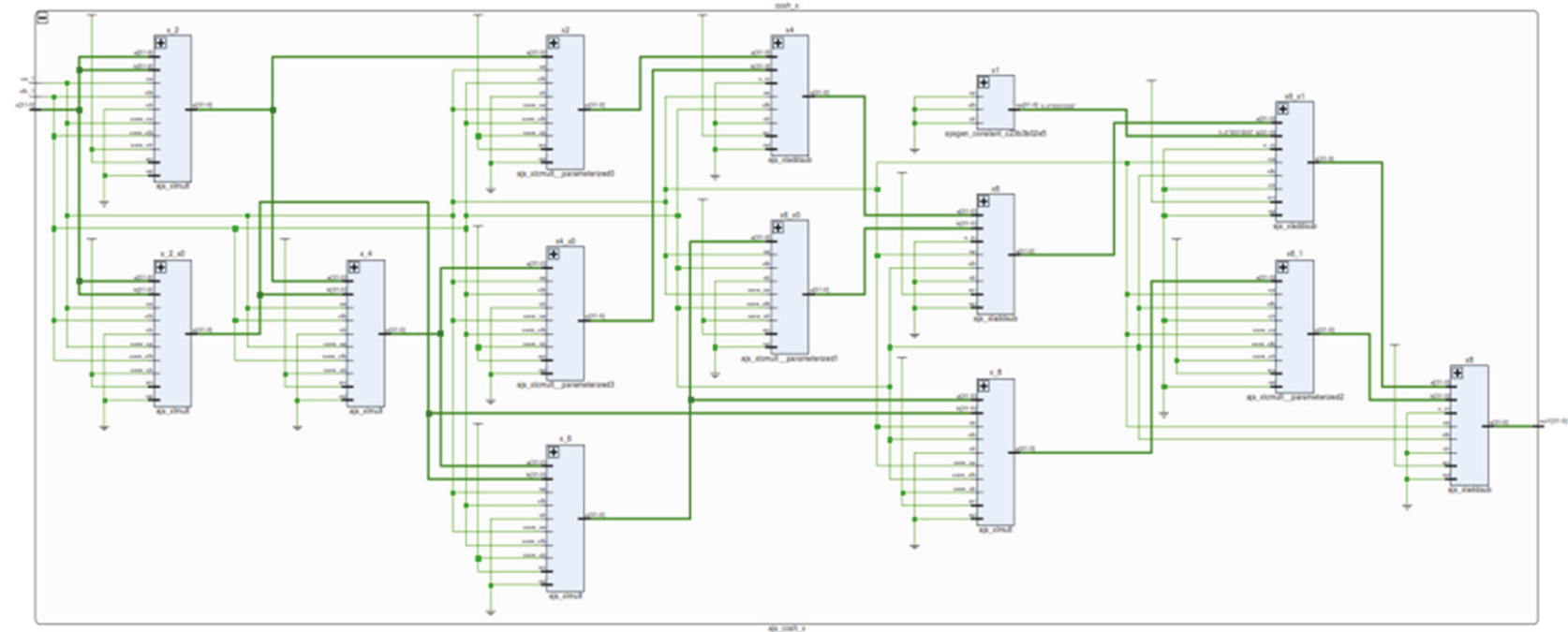

(b)

FIGURE 8: (Color online) RTL schematic of proposed autonomous jerk oscillator implemented in Kintex 7.

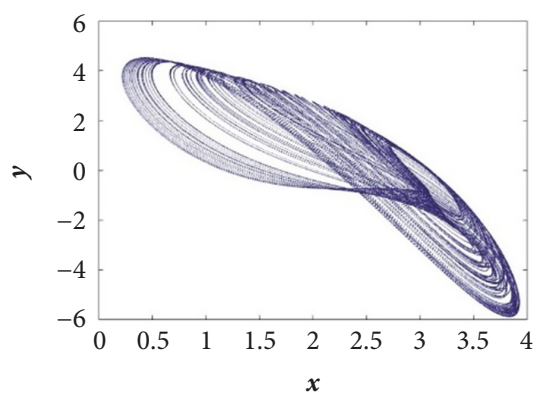

(a)

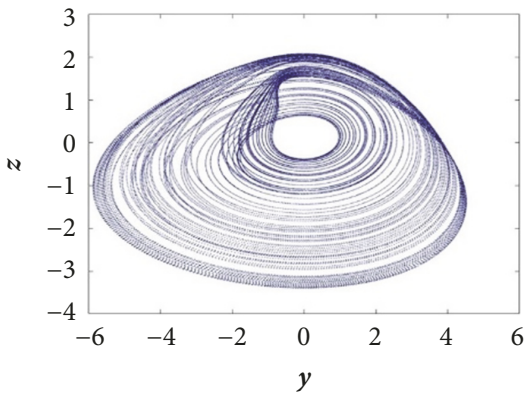

(b)

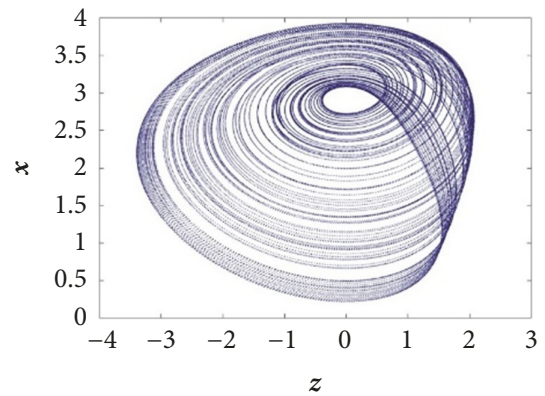

(c)

FIGURE 9: (Color online) Phase portraits of the FPGA implemented of proposed autonomous jerk oscillator with hardware-software cosimulation for parameter values $a=3.3, b=3$ and initial conditions $[1,0,0.5]$ with $h=0.001$. 

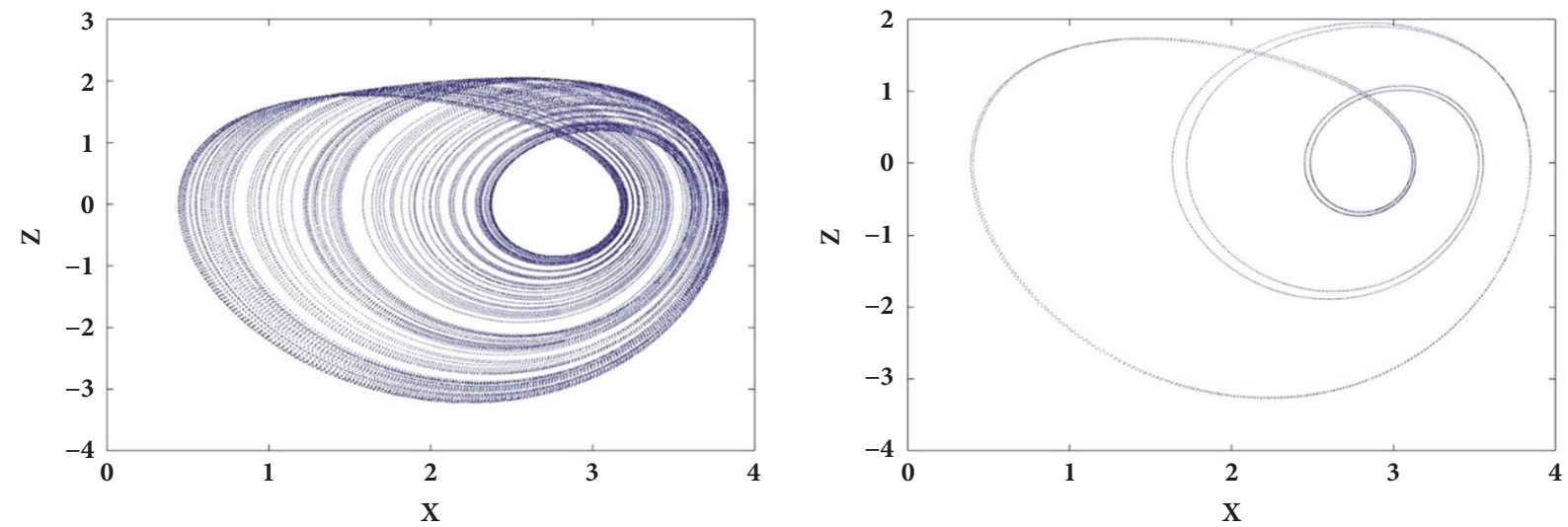

(a)
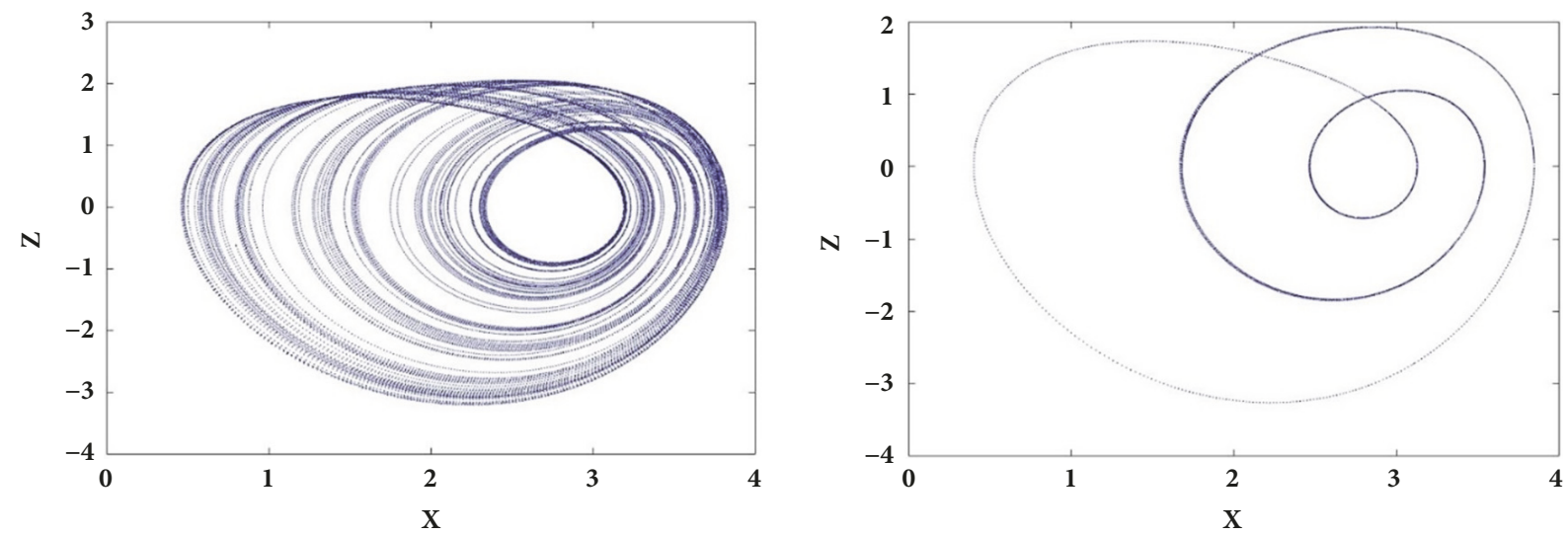

(b)

Figure 10: (Color online) Phase portraits in the plane $(x, z)$ of the FPGA implemented proposed autonomous jerk oscillator for specific values of parameter $b$ : (a) $b=3.185$ and (b) $b=3.189$ for the initial conditions $(\mathrm{x}(0), \mathrm{y}(0), \mathrm{z}(0))=(1.0,0,0.5)$ in left column and $(\mathrm{x}(0)$, $\mathrm{y}(0)$, $\mathrm{z}(0))=(3.0,0,0.5)$ in right column with the value of the parameter $a=3.3$. The step size is taken as $h=0.001$.

The dynamics of the Lyapunov candidate function can be derived as follows:

$$
\dot{V}=s \cdot \dot{s}+(d-\widehat{d})(-\dot{\hat{d}})+(c-\widehat{c})(-\dot{\bar{c}})
$$

Using (17), (16), and (14) in (19),

$$
\begin{aligned}
\dot{V} & \leq k s\left[G\left(x_{s}\right)(d-\widehat{d})-F\left(x_{m}\right)(c-\widehat{c})-\eta \tanh (s)\right. \\
& -\rho s]-(d-\hat{d})(\dot{\vec{d}})-(c-\widehat{c})(\dot{\bar{c}}) .
\end{aligned}
$$

Let us define the parameter estimate laws as

$$
\begin{aligned}
& \dot{\vec{c}}=K_{c} s \cdot G\left(x_{s}\right), \\
& \dot{\vec{d}}=K_{d} s \cdot F\left(x_{m}\right),
\end{aligned}
$$

where $K_{c}, K_{d}$ are positive constants.

Using (21a) and (21b) in (20), we can solve the Lyapunov function dynamics as follows:

$$
\dot{V} \leq-\eta\left|s_{w}\right|-\rho s^{2},
$$

where $\eta$ and $\rho$ are all positive, and $\dot{V}$ is negative definite.
4.2. Numerical Verifications. For numerical validation of the proposed synchronization method, we use the proposed autonomous jerk oscillators as master and slave and apply the adaptive sliding mode control to achieve the synchronization. Let us define the master system as

$$
\begin{aligned}
& \dot{x}_{m}=z_{m}, \\
& \dot{y}_{m}=a x_{m}-y_{m}-b z_{m}-\cosh \left(x_{m}\right), \\
& \dot{z}_{m}=y_{m},
\end{aligned}
$$

where $a, b$ are the system parameters. The slave system with the adaptive sliding mode controllers $\left(u_{i}\right)$ are defined as

$$
\begin{aligned}
& \dot{x}_{s}=z_{s}+u_{x}, \\
& \dot{y}_{s}=a x_{s}-y_{s}-b z_{s}-\cosh \left(x_{s}\right)+u_{y}, \\
& \dot{z}_{s}=y_{s}+u_{z} .
\end{aligned}
$$

The parameters of the slave system are assumed to be unknown with parameter estimates $\widehat{a}, \widehat{b}$. Using the master 


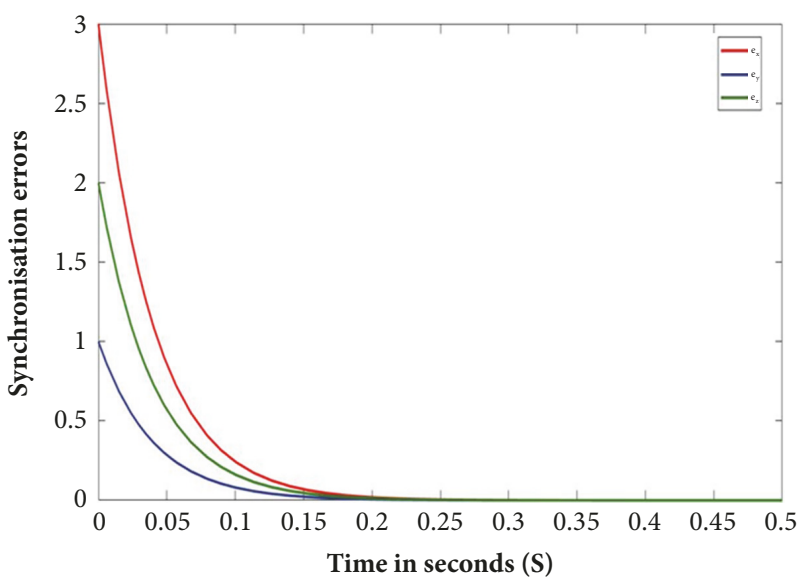

(a)
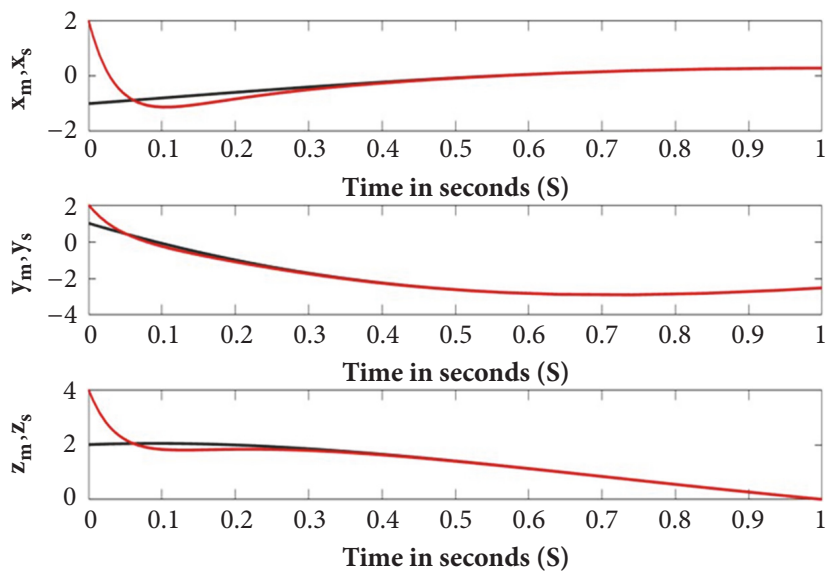

(b)

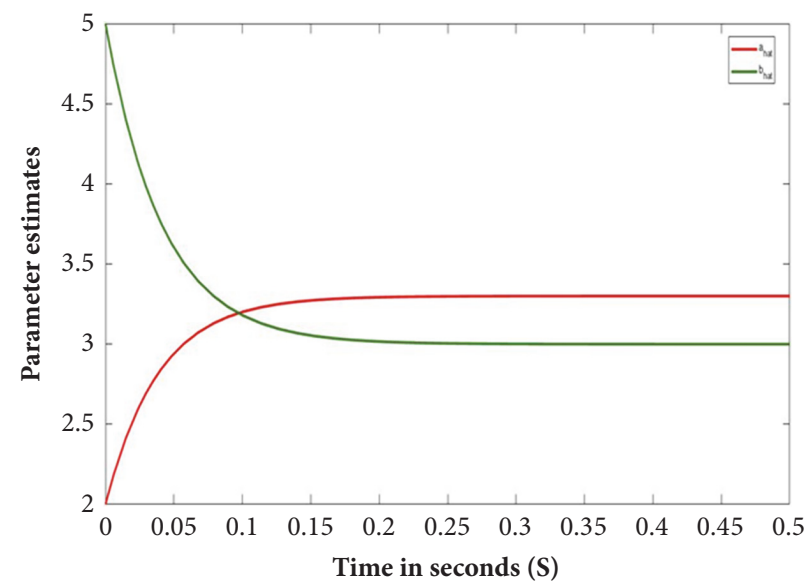

(c)

FIGURE 11: Time history of synchronization error variables (a), synchronized states (b), and estimated parameters (c).

system (23a), (23b), and (23c) and slave system (24a), (24b), and $(24 \mathrm{c})$ in the error dynamics can be derived as follows:

$$
\begin{aligned}
\dot{e}_{x}= & e_{z}+u_{x} \\
\dot{e}_{y}= & \widehat{a} x_{s}-\widehat{b} z_{s}-e_{y}-\cosh \left(x_{s}\right)-a x_{m}+b z_{m} \\
& +\cosh \left(x_{m}\right)+u_{y} \\
& \quad \\
\dot{e}_{z}= & e_{y}+u_{z} .
\end{aligned}
$$

Let the adaptive sliding mode controllers be chosen as follows:

$$
\begin{aligned}
u_{x}= & -e_{z}-\gamma_{x} \tanh \left(s_{x}\right)-\rho_{x} s_{x}-k_{x} e_{x}, \\
u_{y}= & -\widehat{a} e_{x}+\widehat{b} e_{z}+e_{y}+\cosh \left(x_{s}\right)-\cosh \left(x_{m}\right) \\
& -\gamma_{y} \tanh \left(s_{y}\right)-\rho_{y} s_{y}-k_{y} e_{y}, \\
u_{z}= & -e_{y}-\gamma_{z} \tanh \left(s_{z}\right)-\rho_{z} s_{z}-k_{z} e_{z},
\end{aligned}
$$

where $\rho_{i}>0, \gamma_{i}>0$ are the sliding surface gains and $k_{i}$ is the controller gain for $i=x, y, z$
Using (17) and (18) with (13), the parameter update laws can be defined as follows:

$$
\begin{aligned}
& \dot{\hat{a}}=-s_{y} x_{m}, \\
& \dot{\hat{b}}=s_{y} z_{m},
\end{aligned}
$$

where $\dot{\hat{a}}, \dot{\hat{b}}$ are the dynamics of the parameter estimates $\widehat{a}, \widehat{b}$. For numerical simulations, we take the initial conditions of the master system (25a), (25b), and $(25 c)$ as $[-1,1,2]$, slave system as $[2,2,4]$, parameter estimates as $\widehat{a}(0)=$ $2, \widehat{b}(0)=5$, and the proportional constants $K=[1,1,1]$. Figure 11 shows the dynamics of the synchronization errors and synchronized states of the master and slave systems and estimated parameters.

When $t>0.3$, the synchronization error variables converge to zero with exponentially asymptotical speed [see Figure 11(a)] and thereby guaranteeing the synchronization between the master system (23a), (23b), and (23c) and slave system (24a), (24b), and (24c) [see Figure 11(b)]. The unknown parameters of slave system (24a), (24b), and (24c) are simultaneously successfully estimated to their values [see Figure 11(c)]. 


\section{Conclusion}

In this paper, an autonomous jerk oscillator with a cosine hyperbolic nonlinearity and two parameters was proposed and studied. It was demonstrated that the Hopf bifurcation occurs near the equilibrium point as one of jerk oscillator parameter crosses the critical value. The proposed autonomous jerk oscillator exhibits periodic attractors, onescroll chaotic attractors, and coexistence between chaotic and periodic attractors. Period-doubling route to chaos and reverse period-doubling route to chaos was found in proposed autonomous jerk oscillator with the variation of each of its two parameters. Further, the proposed autonomous jerk oscillator was implemented using Field Programmable Gate Array in order to show that the proposed autonomous jerk oscillator is hardware realizable. Finally, synchronization of unidirectional coupled identical proposed autonomous jerk oscillators was achieved using adaptive sliding mode control method.

\section{Data Availability}

No data were used to support this study.

\section{Conflicts of Interest}

The authors declare that there are no conflicts of interest regarding the publication of this paper.

\section{References}

[1] E. N. Lorenz, "Deterministic nonperiodic flow," Journal of the Atmospheric Sciences, vol. 20, no. 2, pp. 130-141, 1963.

[2] A. Tamaševičius, A. Čenys, G. Mykolaitis, A. Namajunas, and E. Lindberg, "Hyperchaotic oscillator with gyrators," IEEE Electronics Letters, vol. 33, no. 7, pp. 542-544, 1997.

[3] M. Lakshmanan and D. V. Senthilkumar, Dynamics of Nonlinear Time-Delay Systems, Springer Series in Synergetics, Springer, Berlin, Germany, 2010.

[4] A. Buscarino, L. Fortuna, M. Frasca, and L. V. Gambuzza, "A chaotic circuit based on Hewlett-Packard memristor," Chaos: An Interdisciplinary Journal of Nonlinear Science, vol. 22, no. 2, Article ID 023136, 2012.

[5] C.-L. Li, H.-M. Li, W. Li et al., "Dynamics, implementation and stability of a chaotic system with coexistence of hyperbolic and non-hyperbolic equilibria," AEÜ - International Journal of Electronics and Communications, vol. 84, pp. 199-205, 2018.

[6] D. Lathrop, Physics Today, vol. 68, no. 4, pp. 54-55, 2015.

[7] B. Muthuswamy and S. Banerjee, "Designing Hardware for FPGAs," in A Route to Chaos Using FPGAs, vol. 16 of Emergence, Complexity and Computation, pp. 29-53, Springer International Publishing, Cham, 2015.

[8] V.-T. Pham, S. Jafari, C. Volos, T. Gotthans, X. Wang, and D. V. Hoang, "A chaotic system with rounded square equilibrium and with no-equilibrium," Optik - International Journal for Light and Electron Optics, vol. 130, pp. 365-371, 2017.

[9] F. Nazarimehr, K. Rajagopal, J. Kengne, S. Jafari, and V.-T. Pham, "A new four-dimensional system containing chaotic or hyperchaotic attractors with no equilibrium, a line of equilibria and unstable equilibria," Chaos, Solitons \& Fractals, vol. 111, pp. 108118, 2018.
[10] J. C. Sprott, “Some simple jerk functions," American Journal of Physics, vol. 65, no. 6, pp. 537-543, 1997.

[11] R. Eichhorn, S. J. Linz, and P. Hänggi, "Simple polynomial classes of chaotic jerky dynamics," Chaos, Solitons \& Fractals, vol. 13, no. 1, pp. 1-15, 2002.

[12] J.-M. Malasoma, "What is the simplest dissipative chaotic jerk equation which is parity invariant?" Physics Letters A, vol. 264, no. 5, pp. 383-389, 2000.

[13] S. H. Schot, "Jerk: the time rate of change of acceleration," American Journal of Physics, vol. 46, no. 11, pp. 1090-1094, 1978.

[14] J. C. Sprott, Elegant Chaos: Algebraically Simple Chaotic Flows, World Scientific, 2010.

[15] M. E. Yalçin, "Multi-scroll and hypercube attractors from a general jerk circuit using Josephson junctions," Chaos, Solitons \& Fractals, vol. 34, no. 5, pp. 1659-1666, 2007.

[16] J. Ma, X. Y. Wu, R. T. Chu, and L. Zhang, "Selection of multiscroll attractors in Jerk circuits and their verification using Pspice," Nonlinear Dynamics, vol. 76, no. 4, pp. 1951-1962, 2014.

[17] J. C. Sprott, "A new chaotic jerk circuit," IEEE Transactions on Circuits and Systems II: Express Briefs, vol. 58, no. 4, pp. 240243, 2011.

[18] C. Volos, A. Akgul, V.-T. Pham, I. Stouboulos, and I. Kyprianidis, "A simple chaotic circuit with a hyperbolic sine function and its use in a sound encryption scheme," Nonlinear Dynamics, vol. 89, no. 2, pp. 1047-1061, 2017.

[19] S. Vaidyanathan, C. Volos, V.-T. Pham, K. Madhavan, and B. A. Idowu, "Adaptive backstepping control, synchronization and circuit simulation of a 3-D novel jerk chaotic system with two hyperbolic sinusoidal nonlinearities," Archives of Control Sciences, vol. 24, no. 3, pp. 375-403, 2014.

[20] A. N. Pisarchik and U. Feudel, "Control of multistability," Physics Reports, vol. 540, no. 4, pp. 167-218, 2014.

[21] X. Luo, M. Small, M.-F. Danca, and G. Chen, "On a dynamical system with multiple chaotic attractors," International Journal of Bifurcation and Chaos, vol. 17, no. 9, pp. 3235-3251, 2007.

[22] Z. T. Njitacke, J. kengne, H. B. Fotsin, A. NguomkamNegou, and D. Tchiotsop, Coexistence of multiple attractors and crisis route to chaos in a novel memristive diode bidge-based Jerk circuit, Chaos, Solitons \& Fractals, vol. 99, 2017.

[23] B.-C. Bao, Q. Xu, H. Bao, and M. Chen, "Extreme multistability in a memristive circuit," IEEE Electronics Letters, vol. 52, no. 12, pp. 1008-1010, 2016.

[24] B. Bao, T. Jiang, Q. Xu, M. Chen, H. Wu, and Y. Hu, "Coexisting infinitely many attractors in active band-pass filter-based memristive circuit," Nonlinear Dynamics, vol. 86, no. 3, pp. 1711-1723, 2016.

[25] A. Massoudi, M. G. Mahjani, and M. Jafarian, "Multiple attractors in Koper-Gaspard model of electrochemical periodic and chaotic oscillations," Journal of Electroanalytical Chemistry, vol. 647, no. 1, pp. 74-86, 2010.

[26] V.-T. Pham, C. Volos, S. Jafari, and T. Kapitaniak, "Coexistence of hidden chaotic attractors in a novel no-equilibrium system," Nonlinear Dynamics, vol. 87, no. 3, pp. 2001-2010, 2017.

[27] Z. T. Njitacke, J. Kengne, N. A. Nguomkam, T. M. Fouodji, and H. B. Fotsin, "Coexistence of multiple attractorsand crisis route to chaos in a novel chaotic jerk circuit," International Journal of Bifurcation and Chaos, vol. 25, pp. 1550052-1550060, 2015.

[28] J. Kengne, Z. T. Njitacke, and H. B. Fotsin, "Dynamical analysis of a simple autonomous jerk system with multiple attractors," Nonlinear Dynamics, vol. 83, no. 1-2, pp. 751-765, 2016. 
[29] J. Kengne, A. Nguomkam Negou, and Z. T. Njitacke, "Antimonotonicity, chaos and multiple attractors in a novel autonomous jerk circuit," International Journal of Bifurcation and Chaos, vol. 27, no. 7, 1750100, 20 pages, 2017.

[30] Z. T. Njitacke, J. kengne, and L. Kamdjeu Kengne, "Antimonotonicity, chaos and multiple coexisting attractors in a simple hybrid diode-based jerk circuit," Chaos, Solitons \& Fractals, vol. 105, pp. 77-91, 2017.

[31] J. Kengne, A. N. Negou, and D. Tchiotsop, "Antimonotonicity, chaos and multiple attractors in a novel autonomous memristor-based jerk circuit," Nonlinear Dynamics, vol. 88, no. 4, pp. 2589-2608, 2017.

[32] E. Tlelo-Cuautle, A. D. Pano-Azucena, J. J. Rangel-Magdaleno, V. H. Carbajal-Gomez, and G. Rodriguez-Gomez, "Generating a 50-scroll chaotic attractor at $66 \mathrm{MHz}$ by using FPGAs," Nonlinear Dynamics, vol. 85, no. 4, pp. 2143-2157, 2016.

[33] K. Rajagopal, A. Akgul, S. Jafari, A. Karthikeyan, and I. Koyuncu, "Chaotic chameleon: Dynamic analyses, circuit implementation, FPGA design and fractional-order form with basic analyses," Chaos, Solitons \& Fractals, vol. 103, pp. 476-487, 2017.

[34] E. Dong, Z. Liang, S. Du, and Z. Chen, “Topological horseshoe analysis on a four-wing chaotic attractor and its FPGA implement," Nonlinear Dynamics, vol. 83, no. 1-2, pp. 623-630, 2016.

[35] S. M. Ismail, L. A. Said, A. A. Rezk et al., "Generalized fractional logistic map encryption system based on FPGA," AE ̈̈ - International Journal of Electronics and Communications, vol. 80, pp. 114-126, 2017.

[36] E. Tlelo-Cuautle, J. J. Rangel-Magdaleno, A. D. Pano-Azucena, P. J. Obeso-Rodelo, and J. C. Nunez-Perez, "FPGA realization of multi-scroll chaotic oscillators," Communications in Nonlinear Science and Numerical Simulation, vol. 27, no. 1-3, pp. 66-80, 2015.

[37] Y.-M. Xu, L.-D. Wang, and S.-K. Duan, "A memristor-based chaotic system and its field programmable gate array implementation," Acta Physica Sinica, vol. 65, no. 12, Article ID 120503, 2016.

[38] E. Tlelo-Cuautle, L. G. de la Fraga, V.-T. Pham, C. Volos, S. Jafari, and A. D. J. Quintas-Valles, "Dynamics, FPGA realization and application of a chaotic system with an infinite number of equilibrium points," Nonlinear Dynamics, vol. 89, no. 2, pp. 1129-1139, 2017.

[39] Z. Wei, A. Akgul, U. E. Kocamaz, I. Moroz, and W. Zhang, Control, electronic circuit application and fractional-order analysis of hidden chaotic attractors in the self-exciting homopolar disc dynamo, Chaos, Solitons \& Fractals, vol. 111, 2018.

[40] D. Valli, B. Muthuswamy, S. Banerjee et al., "Synchronization in coupled Ikeda delay systems," The European Physical Journal Special Topics, vol. 223, no. 8, pp. 1465-1479, 2014.

[41] Karthikeyan Rajagopal, Anitha Karthikeyan, and Prakash Duraisamy, "Hyperchaotic Chameleon: Fractional Order FPGA Implementation," Complexity, vol. 2017, Article ID 8979408, 16 pages, 2017.

[42] Karthikeyan Rajagopal, Laarem Guessas, Sundarapandian Vaidyanathan, Anitha Karthikeyan, and Ashokkumar Srinivasan, "Dynamical Analysis and FPGA Implementation of a Novel Hyperchaotic System and Its Synchronization Using Adaptive Sliding Mode Control and Genetically Optimized PID Control," Mathematical Problems in Engineering, vol. 2017, Article ID 7307452, 14 pages, 2017.

[43] Karthikeyan Rajagopal, Laarem Guessas, Anitha Karthikeyan, Ashokkumar Srinivasan, and Girma Adam, "Fractional Order
Memristor No Equilibrium Chaotic System with Its Adaptive Sliding Mode Synchronization and Genetically Optimized Fractional Order PID Synchronization," Complexity, vol. 2017, Article ID 1892618, 19 pages, 2017.

[44] K. Rajagopal, A. Karthikeyan, and A. K. Srinivasan, "FPGA implementation of novel fractional-order chaotic systems with two equilibriums and no equilibrium and its adaptive sliding mode synchronization," Nonlinear Dynamics, vol. 87, no. 4, pp. 2281-2304, 2017.

[45] J. M. Bahi, X. Fang, C. Guyeux, and L. Larger, "FPGA design for pseudorandom number generator based on chaotic iteration used in information hiding application," Applied Mathematics \& Information Sciences, vol. 7, no. 6, pp. 2175-2188, 2013.

[46] C. Chen, H. Ma, H. Chen, Y. Meng, and Q. Ding, "FPGA implementation of a UPT chaotic signal generator for image encryption," Pacific Science Review A: Natural Science and Engineering, vol. 17, no. 3, pp. 97-102, 2015.

[47] M. L. Barakat, A. G. Radwan, and K. N. Salama, "Hardware realization of chaos based block cipher for image encryption," in Proceedings of the 2011 23rd International Conference on Microelectronics, ICM 2011, Tunisia, December 2011.

[48] A. M. Atteya and A. H. Madian, "A hybrid Chaos-AES encryption algorithm and its impelmention based on FPGA," in Proceedings of the 2014 12th IEEE International New Circuits and Systems Conference, NEWCAS 2014, pp. 217-220, Canada, June 2014.

[49] S. T. Kingni, V.-T. Pham, S. Jafari, and P. Woafo, "A chaotic system with an infinite number of equilibrium points located on a line and on a hyperbola and its fractional-order form," Chaos, Solitons \& Fractals, vol. 99, pp. 209-218, 2017.

[50] B. A. Idowu, U. E. Vincent, and A. N. Njah, "Synchronization of chaos in non-identical parametrically excited systems," Chaos, Solitons \& Fractals, vol. 39, no. 5, pp. 2322-2331, 2009.

[51] V. Sundarapandian and R. Karthikeyan, "Anti-synchronization of Lü and Pan chaotic systems by adaptive nonlinear control," European Journal of Scientific Research, vol. 64, no. 1, pp. 94106, 2011.

[52] V. Sundarapandian and R. Karthikeyan, "Adaptive anti-synchronization of Uncertain Tigan and Li Systems," Journal of Engineering and Applied Sciences, vol. 7, no. 1, pp. 45-52, 2012.

[53] S. S. Majidabad and H. T. Shandiz, "Discrete-time Terminal Sliding Mode Control of Chaotic Lorenz System," Journal of Control and Systems Engineering, vol. 1, no. 1, pp. 1-8, 2013.

[54] S. Vaidyanathan, "A new 3-D jerk chaotic system with two cubic nonlinearities and its adaptive backstepping control," Archives of Control Sciences. Polish Academy of Sciences. Committee of Automatic Control and Robotics, vol. 27(63), no. 3, pp. 409-439, 2017.

[55] O. S. Onma, O. I. Olusola, and A. N. Njah, "Control and Synchronization of Chaotic and Hyperchaotic Lorenz Systems via Extended Backstepping Techniques," Journal of Nonlinear Dynamics, vol. 2014, Article ID 861727, 15 pages, 2014.

[56] B. Wang, Y. Li, and D. L. Zhu, "Simplified sliding mode of a novel class of four-dimensional fractional-order chaos," International Journal of Control and Automation, vol. 8, no. 8, pp. 425-438, 2015.

[57] C. Yin, S. Dadras, S.-M. Zhong, and Y. Chen, "Control of a novel class of fractional-order chaotic systems via adaptive sliding mode control approach," Applied Mathematical Modelling, vol. 37, no. 4, pp. 2469-2483, 2013.

[58] H. Liu and J. Yang, "Sliding-mode synchronization control for uncertain fractional-order chaotic systems with time delay," Entropy, vol. 17, no. 6, pp. 4202-4214, 2015. 
[59] S. Wang, Y. G. Yu, and M. Diao, "Hybrid projective synchronization of chaotic fractional order systems with different dimensions," Physica A: Statistical Mechanics and its Applications, vol. 389, no. 21, pp. 4981-4988, 2010.

[60] J. Slotine and W. Li, Applied Nonlinear Control, Prentice Hall, New Jersey, NJ, USA, 1991. 


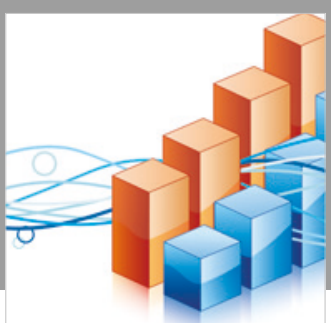

Advances in

Operations Research

\section{-n-m}
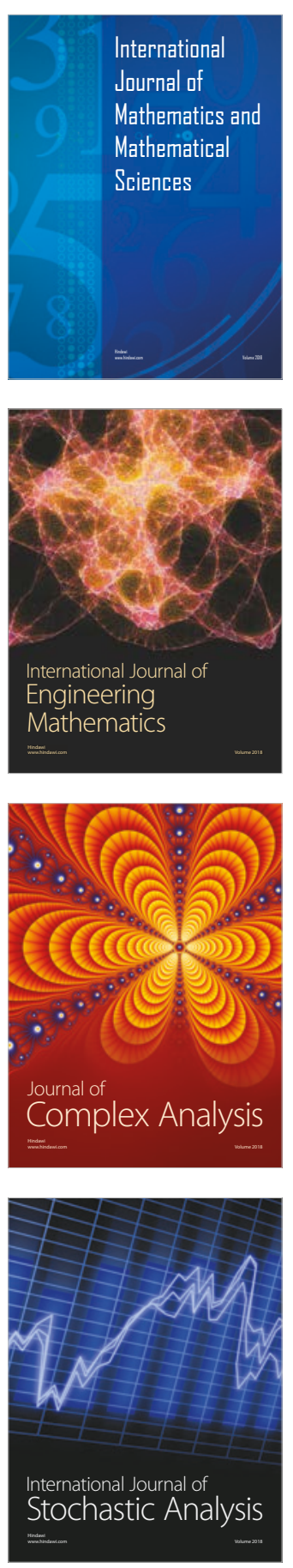
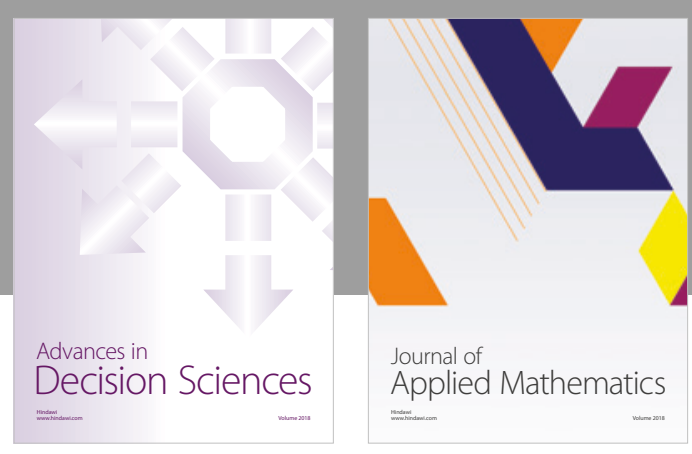

Journal of

Applied Mathematics
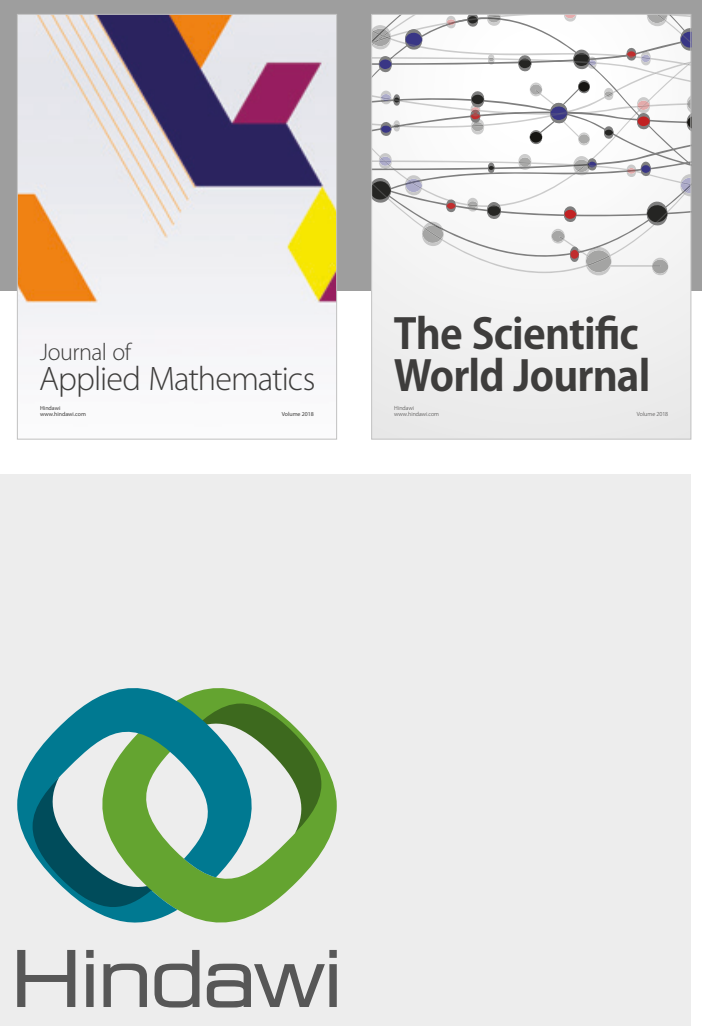

Submit your manuscripts at

www.hindawi.com

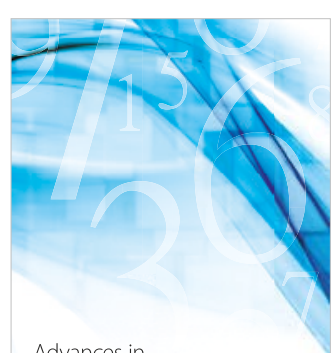

Advances in
Numerical Analysis
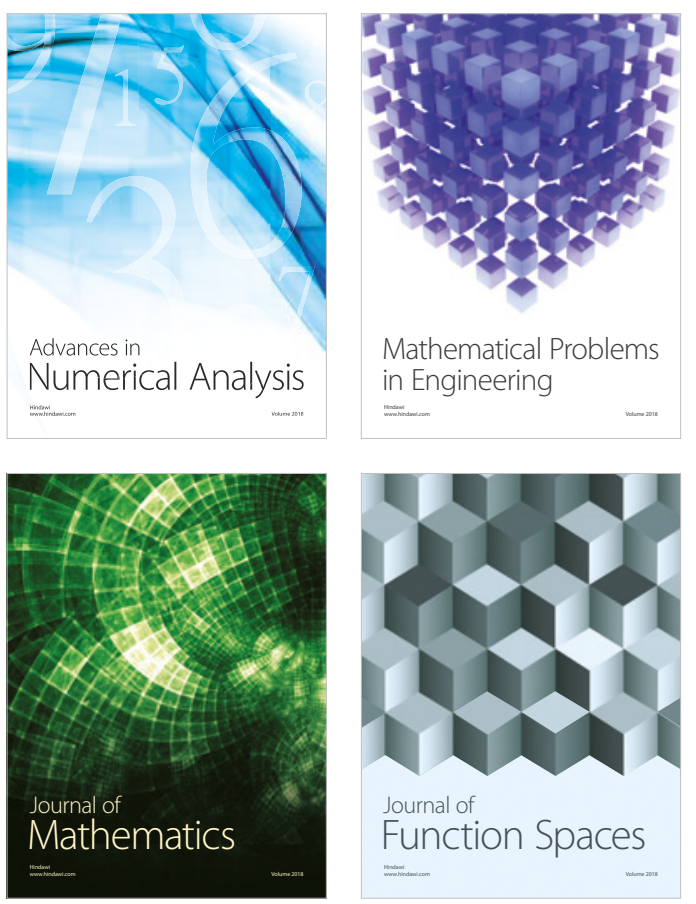

Mathematical Problems in Engineering

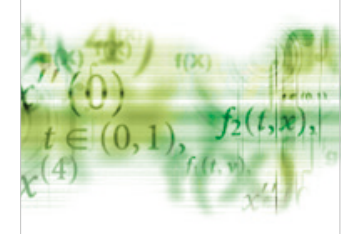

International Journal of

Differential Equations

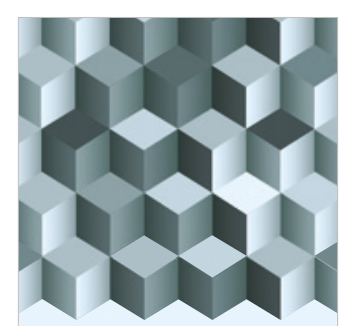

Journal of

Function Spaces

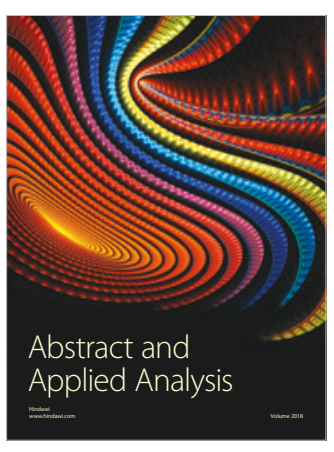

The Scientific

World Journal

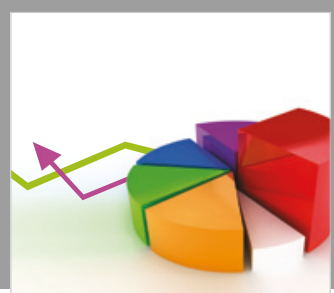

Journal of

Probability and Statistics
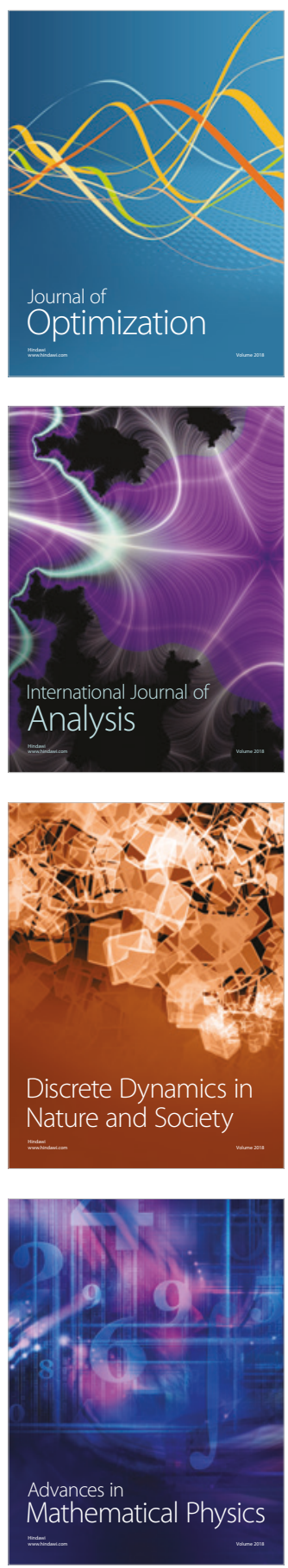